\title{
facial Emotion Recognition Using Average face Ratios AND FuZZY HAMMING DISTANCE
}

\section{Khalid Ounachad, Mohamed Oualla, Abdelalim Sadiq, Abdelghani Souhar}

\section{DOI: $10.14313 / J A M R I S / 4-2020 / 43$}

\begin{abstract}
Facial emotion recognition (FER) is an important topic in the fields of computer vision and artificial intelligence owing to its significant academic and commercial potential. Nowadays, emotional factors are important as classic functional aspects of customer purchasing behavior. Purchasing choices and decisions making are the result of a careful analysis of the product advantages and disadvantages and of affective and emotional aspects. This paper presents a novel method for human emotion classification and recognition. We generate seven referential faces suitable for each kind of facial emotion based on perfect face ratios and some classical averages. The basic idea is to extract perfect face ratios for emotional face and for each referential face as features and calculate the distance between them by using fuzzy hamming distance. To extract perfect face ratios, we use the point landmarks in the face then sixteen features will be extract. An experimental evaluation demonstrates the satisfactory performance of our approach on WSEFEP dataset. It can be applied with any existing facial emotion dataset. The proposed algorithm will be a competitor of the other proposed relative approaches. The recognition rate reaches more than $90 \%$.
\end{abstract}

Keywords: Average Face Ratios, Facial Emotion Recognition, Fuzzy Hamming distance, Perfect Face Ratios

\section{Introduction}

People's face is the most exposed part of body. Emotion is one of the most important attributes of face. Emotions are used in the process of non-verbal communication. They help us to understand the interactions of others. People, can immediately recognize an emotional state of a person. They are seven basics kinds of emotions: Anger, Disgust, Fear, Happiness, Neutral, Sadness and Surprise [1]. The accurate emotion recognition enables you to perform emotion based, sophisticated tasks, such as advertising customers satisfaction analysis.

Face Emotion Recognition systems (FER) aim to recognize a face emotion in a dataset of photos images. The task of emotion recognition is particularly difficult: There doesn't exist a large dataset of training images and classifying emotion can be also difficult. In a face emotion recognition system, the feature extraction is the core block. Matching is used to recognize the right kind of emotion using the precomputed features. The preprocessing step can boost the final performance of the system considerably. Feature extraction aims to transform the input face emotion image into a set of features. Matching is a general concept to describe the task of finding correspondences between two elements that has to be carried out for recognition or classification. It can attempt a simple comparison between the features extracted or more complex comparison systems by using some distances.

The objective of this paper is to propose our method based on fuzzy hamming distance with average face ratios to recognizing a face emotion. This paper presents a novel method for human emotion classification and recognition. We generate seven referential faces suitable for each kind of facial emotion based on perfect face ratios and five classical averages: arithmetic mean, geometric mean, harmonic mean, contraharmonic mean and quadratic mean. The basic idea is to extract perfect face ratios for emotional face and for each referential face as features and calculate the distance between them by using fuzzy hamming distance. To extract perfect face ratios, we use the point landmarks in the face then sixteen features will be extract. To compute distances between the input facial emotion image and each image in a set of our referential faces, the hamming distance based on logical exclusive-or (XOR) function is used.

The Hamming distance evaluates the number of bits that differ from two binary vectors. The Fuzzy Hamming distance [2] has been published to solve Hamming distance limitations on real numbers. This distance is used in this work because real values features are computed, not binary numbers. It ensures great performances in terms of speed and accuracy.

This article is organized as follows. Section 2 related works and background information about four classical averages, perfect face ratios and fuzzy hamming distance, their formalisms and their definitions, section 3, the proposed architecture of our approach is explained in depth. Experimental results are given in section 4 . In section 5 , a conclusion is presented.

\section{Related Works}

Over the last decades, there has been a wealth of research in hamming distance, as well as in its ap- 
plication in computer vision [10] especially in Face Sketch recognition [3], in Banknote Validator [4] and in Content-Based Image Retrieval System (CBIRS) [5]:

- In [3] we presented a new facial sketch recognition method based on fuzzy hamming distance with geometric relationships (face ratios). We proposed to simplify the procedure based on fuzzy hamming distance to use only vectors with simple real values of characteristics. An interesting contribution of [3] is that can accurately recognize the photo of the sketch's face. The proposed algorithm will be a competitor of the other proposed relative approaches. The recognition rate reaches $100 \%$ especially in the CUHK dataset.

- The Fuzzy Hamming Distance based approach, proposed in Banknote Validator [4] and combines the versatility of an automatic system with basic banknote specific information. Subsequently, the system can be updated to use in-depth security features provided by an expert. Fuzzy Hamming distance is used to measure the similarity between banknotes

- The study suggested by M. Ionescu et al. [5, 6] presented initial results on a new approach to measure similarity between images using the notion of Fuzzy Hamming Distance (FHD) and its use to CBIR. The main advantage of the FHD is that the extent to which two different images are considered indeed different can be tuned to become more context dependent and to capture (implicit) semantic image information. The study shows good results using complete linkage agglomerative clustering. Fuzzy Hamming Distance proved to be efficient in a Content Based Image Retrieval system that output the closest images in the database given a query image. In [6], the obtained results for image retrieval based on using hamming distance showed effectiveness of their approach.

- In [7], character recognition is carried out using template-matching scheme using correlation. In their approach, in addition to correlation, the hamming distance was applied in the scenarios where the correlation fails, the text image is segmented into lines and then characters by basic pre-processing techniques. After that, the character image is classified and converted into text by template matching scheme using either correlation or hamming distance. For test stage, four text images were created with total of 560 characters. Their system works successfully with average recognition rates of $72.39 \%$ and $94.90 \%$ for correlation only and correlation plus hamming distance, respectively.

Contribution. This article proposes a new facial emotion recognition method based on fuzzy Hamming distance with face ratios and their geometric relationships. Our work is inspired in part by the recent and successful method that has shown that relatively simple benchmark features could be used to perform well in a fuzzy Hamming distance-based face emotion recognition framework. In this paper, we simplify the procedure based on fuzzy hamming distance to use only vectors with simple real values of characteristics. A key technical contribution of our paper is a method for recognizing a facial emotion based on these simple features that can accurately recognize the right kind of the emotion's face. This method achieves our goal by producing a recognition rate reaches more than $93 \%$.

\section{Background Information}

Symbolically, we have a data set containing the values $x_{1}, x_{2}, \ldots, X_{n}$.

The arithmetic mean (Average) is equal to the sum of all numerical values of a set divided by the number of items in that set, then the average $\mathrm{A}$ is defined by the formula:

$$
A=\frac{1}{n} \sum_{i=1}^{n} X_{i}=\frac{s}{N}
$$

$N=$ The number of items being averaged.

$S=$ The sum of the numbers being averaged.

The geometric mean is the nth root of the product of $n$ numbers, for a set of numbers, then the average $G$ is defined by the formula:

$$
G=\left(\prod_{i=1}^{n} x_{i}\right)^{\frac{1}{n}}=\sqrt[n]{X_{1} X_{2} \ldots X_{n}}
$$

The harmonic mean is the reciprocal of the arithmetic mean of the reciprocals of the given numbers, then the average $\mathrm{H}$ is defined by the formula:

$$
H=\frac{n}{\sum_{i=1}^{n} \frac{1}{x_{i}}}
$$

The contraharmonic mean is the arithmetic mean of the squares of the values divided by the arithmetic mean of tge values, then the average $C$ is defined by the formula:

$$
C=\frac{\frac{x_{1}^{2}+x_{2}^{2}+\ldots+x_{n}^{2}}{n}}{\frac{x_{1}+x_{2} \ldots+x_{n}}{n}}=\frac{x_{1}^{2}+x_{2}^{2}+\ldots+x_{n}^{2}}{x_{1}+x_{2} \ldots+x_{n}}
$$

The quadratic mean is calculated as the square root of the mean of the of the given numbers, then the average $Q$ is defined by the formula:

$$
Q=\sqrt{\frac{x_{1}^{2}+x_{2}^{2}+\ldots+x_{n}^{2}}{n}}
$$

Let is a finite field with q elements.

The Hamming distance [9] d (x,y) between two vectors $x, y \in F(n)$ is the number of coefficients in which they differ,

$$
d(x, y)=\operatorname{Card}\left\{i / x_{i} \neq y_{i}\right\}
$$


The Degree of difference [9]: Given the real values $x$ and $y$, the degree of difference between $x$ and $y$, modulated by $\alpha>0$, denoted by $d_{\alpha}(x, y)$ is defined as:

$$
d_{\alpha}(x, y)=1-e^{-\alpha(x-y)^{2}}
$$

The parameter $\alpha$ modulates the degree of difference in the sense that for the same value of $|x-y|$ different values of will result in different values of $d_{\alpha}(x, y)$

The Difference fuzzy set for two vectors [9]

Let $x$ and $y$ be two-dimensional real vectors, and let $x i, y i$ denote their corresponding ith component. The degree of difference between $x$ and $y$ along the component $i$, modulated by the parameter $\alpha$ is $d_{\alpha}$ (xi,yi). The difference fuzzy set corresponding to $d_{\alpha}(x i, y i)$ is $D_{\alpha}(x i, y i)$ with membership function:

$$
\begin{aligned}
& \mu_{D_{\alpha}}(x, y)(i):\{1, \ldots, n\} \rightarrow[0,1] \\
& \mu D_{\alpha}(x, y)(i)=d_{\alpha}(x i, y i)
\end{aligned}
$$

$\mu D_{\alpha}(x, y)(i)$ is the degree to which the vectors and are different along there component.

The Cardinality of a fuzzy set [9]:

Let: $A \equiv \sum_{i=1}^{n} x i / \mu i$

denote the discrete fuzzy set $A$ over the universe of discourse $\{x 1, \ldots, x n\}$ where $\mu i=\mu A(x i)$ denotes the degree of membership for $x i$ to $A$. The cardinality, $\operatorname{Card} A$, of $A$ is a fuzzy set:

$$
\operatorname{Card} A \equiv \sum_{i=1}^{n} \frac{i}{\mu_{\operatorname{Card}(A)}(i)}
$$

where: $\mu_{\operatorname{Card}(A)=\mu_{(i)} \wedge\left(1-\mu_{(i+1)}\right)}$

and where $\mu_{(i)}$ denotes the ith largest value of $\mu \mathrm{i}$, the values

$\mu_{(0)}=1$ and $\mu_{\left(n_{+1}\right)}=0$ are introduced for convenience, and ${ }^{\wedge}$ denotes the min operation.

the non-fuzzy cardinality $n \operatorname{Card}(A)$, is:

$$
n \operatorname{Card}(A)=\operatorname{card} \overline{\left(\left\{x ; \mu_{(A)}(x)>0.5\right\}\right)}
$$

Where for a set $S, \bar{S}$ denotes the closure of .

The fuzzy Hamming distance [9] between $x$ and $y$, denoted by $F H D_{\alpha}(x, y)$ is the fuzzy cardinality of the difference fuzzy set, $D_{\alpha}(x, y)$ :

$$
\begin{aligned}
& \mu_{F H D(x, y)}(. \alpha):\{1, \ldots, n\} \rightarrow[0,1] \\
& \mu_{F H D(x, y)}(k, \alpha)=\mu_{\operatorname{Card}_{\alpha}(x, y)}(k)
\end{aligned}
$$

For $k \in\{1, \ldots, n\}$ where $n=\left|\operatorname{Support}_{\alpha}(x, y)\right|$ for a given value $k, \mu_{F H D(x, y)}(k, \alpha)$ is the degree to which the vectors and are different on exactly $k$ components (with the modulation constant $\alpha$ ).

Based on the rules used to choose the perfect face, based on the ideas of the famous Italian doctor one of the founder of criminology and based also on the ideal proportions of the face described by rhino plastic doctors, the ratios of the distances taken as the scientific measuring guns of the beauty in a perfect face are calculated. the same distances are computed for each face sketch in dataset of the face sketches.

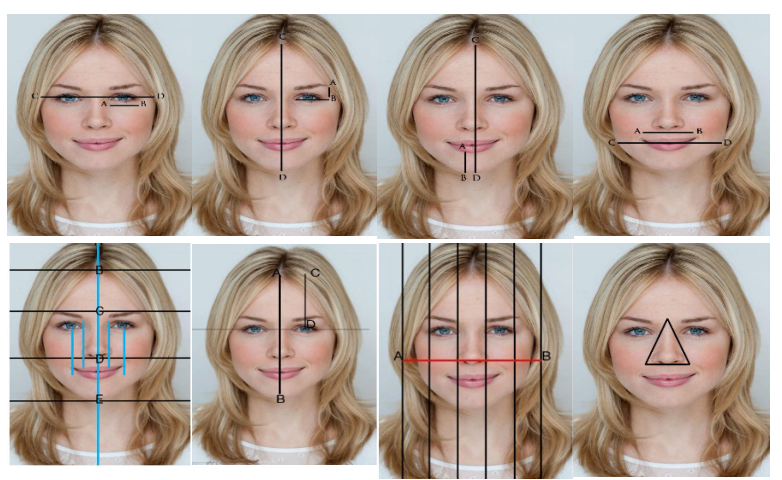

Fig. 1. Photos of Colgate, an 18-year-old Briton, won a beauty pageant where the contestants were judged on scientific criteria. The scientifcs rules used from left to right and from top to bottom: $A B=1 / 5 C D$, $A B=1 / 10 C D, A B=1 / 5 C D, A B=1 / 2 C D, B C=C D=D E=1 / 3 B E$; $A B=1 / 2 B C, C D=1 / 2 A B, A B=5$ eyes, nose surface $<=5 \%$ total of facial surface

Fig. 1 illustrated the scientific rules used to select the beauty queen.

The different distances used to calculate the

proportions of the face are inspired by cannons in a perfect face. We define the following distances as:

d0: $\quad$ The face width

d1: The distance between the end of the right eye and the right end of the face

d2: The length of the right eye

d3: The distance between the eyes

d4: The length of the left eye

d5: The distance between the end of the left eye and the left end of the face

d6: The distance between the centers of the pupils

d7: The mouth length

d8: The distance between eyes

d9: The nose width

d10: The mouth length

d11: The jaw length

d12: The distance between the eyes and the last point of the head

d13: The distance between the eyes and the chin

d14: distance between the center of the forehead and the last point of the head

d15: The distance between the center of the forehead and the nose

d16: The distance between nose and the chin

d17: The distance between the eye and the eyebrow

d18: The length of one eye

d19: The distance between the low lip and the chin

d20: The facial length 


\section{Approach}

Our system has two modes, in them, the input facial emotion photo and all faces emotion photos of the dataset are converted to a Gray level, they are resized and cropped into $200 \times 250$ pixels. These dimensions are chosen: It's the proposed default choice of the datasets used and it's also the dimensions used in related works. The Haar-cascades [11] are used for detecting the face in each photo image. The first step of the system is to pretrain and to normalize all the emotional photos in the offline phase. For that they have been transformed into a gray level image and are all cropped to $200 \times 250$ pixels. The same technique is thus used to online mode. After this step, we projected the famous algorithm of viola and jones to detect the faces of the images. The process that follows this second step is used to locate the 68_point_landmarks [8] in each face. These 68 points will be the parameter of our descriptor, which allows to extract an identity of each face via the calculation of the rations of the perfect face. A vector will be dedicated to group these harmonious distances in order. This vector represents a real proportionality with any other similar vector. The ratios of these distances in the vector have been stored as already detailed in this section.

An overview of our proposed Fuzzy Hamming Distance based framework for face sketch recognition is shown in Fig. 2.

In online process of the Face Emotion Recognition System, given a facial emotion, sixteen features are extracted. The series of these characteristics composes a vector of real values. This vector is considered as an identifier of the face from which the values have been extracted and calculated.

In offline process of the Facial Emotion Recognition System, we grouped the dataset of facial emotion photos into seven data subsets based on the kind of emotion that it represents. The same distances (used in online process) are extracted and calculated for each facial emotion photo for each data subset in the facial emotion dataset. We generate seven referential faces suitable for each kind of facial emotion based on the classical means or averages: Arthritic mean, Contra harmonic mean, Geometric mean, Harmonic mean and quadratic mean. We use the landmarks points the generate all referential faces.

After such a facial emotion photos and referential faces transformation to vectors of real values, Facial emotion recognition becomes straightforward. We can compare the facial emotion with the referential facials emotions using the Fuzzy Hamming Distance. In fact, we first compute the landmarks points for each facial emotion image and also for all referential faces. The face ratios for each one is then used as feature vectors for final classification and recognition. lows.

The detail algorithm can be summarized as fol-

1. To pretrain and to normalize all the facial emotion photos

2. Extract the landmarks points of the facial emotion photos according to the model "The 68_face_landmarks"
3. Calculate the distances $\mathrm{d} 0$ to $\mathrm{d} 20$ as defined in section 3.

we assume that:

$$
\begin{array}{llll}
x_{1}=\frac{d 1}{d 0} & x_{2}=\frac{d 2}{d 0} & x_{3}=\frac{d 3}{d 0} & x_{4}=\frac{d 4}{d 0} \\
x_{5}=\frac{d 5}{d 0} & x_{6}=\frac{d 6}{d 7} & x_{7}=\frac{d 8}{d 9}, & x_{8}=\frac{d 10}{d 11} \\
x_{9}=\frac{d 12}{d 13} & x_{10}=\frac{d 14}{d 20} & x_{11}=\frac{d 15}{d 20} & x_{12}=\frac{d 16}{d 20} \\
x_{13}=\frac{d 17}{d 18} & x_{14}=\frac{d 19}{d 20} & &
\end{array}
$$

4. Calculate S: the surface of the nose (Fig. 2)

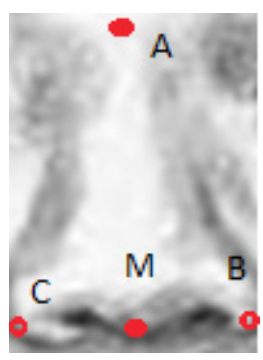

Fig. 2. The principle used for the calculation of the surface of the nose $S$ is based on the Manhattan distance used here to calculate the sides of the triangle encompassing the nose

The Manhattan distance is used here to calculate the sides of the triangle encompassing the nose.

$\mathrm{S}=\frac{1}{4} \sqrt{(\mathrm{a}+\mathrm{b}+\mathrm{c})(\mathrm{a}+\mathrm{b}-\mathrm{c})(-\mathrm{a}+\mathrm{b}+\mathrm{c})(\mathrm{a}-\mathrm{b}+\mathrm{c})}$

we define: $\mathrm{x}_{15}=\mathrm{S}$.

5. Let:

$\mathrm{E}=$ The tragus center of the ear

I= Center inter eyebrow

$\mathrm{F}=$ Center of the front

$\mathrm{C}=$ The chin

$\mathrm{N}=$ Dorsum of nose

The following angles are calculated:

$$
\begin{aligned}
& \theta 1=\overline{\mathrm{FEI}}=\frac{\arccos \left(\mathrm{EF}^{2}+\mathrm{EI}^{2}-\mathrm{FI}^{2}\right)}{2 \times \mathrm{EF} \times \mathrm{EI}} \\
& \theta 2=\overline{\mathrm{IEN}}=\frac{\arccos \left(\mathrm{EN}^{2}+\mathrm{EI}^{2}-\mathrm{NI}^{2}\right)}{2 \times \mathrm{EN} \times \mathrm{EI}} \\
& \theta 3=\overline{\mathrm{NEC}}=\frac{\arccos \left(\mathrm{EN}^{2}+\mathrm{EC}^{2}-\mathrm{NC}^{2}\right)}{2 \times \mathrm{EN} \times \mathrm{EC}}
\end{aligned}
$$

$\mathrm{x}_{16}=\frac{\theta 1+\theta 2}{\theta 3}$

Then:

$$
\mathrm{x}_{16}=\frac{\frac{\arccos \left(\mathrm{EF}^{2}+\mathrm{EI}^{2}-\mathrm{FI}^{2}\right)}{2 \times \mathrm{EF} \times \mathrm{EI}}+\frac{\arccos \left(\mathrm{EN}^{2}+\mathrm{EI}^{2}-\mathrm{NI}^{2}\right)}{2 \times \mathrm{EN} \times \mathrm{EI}}}{\frac{\arccos \left(\mathrm{EN}^{2}+\mathrm{EC}^{2}-\mathrm{NC}^{2}\right)}{2 \times \mathrm{EN} \times \mathrm{EC}}}
$$




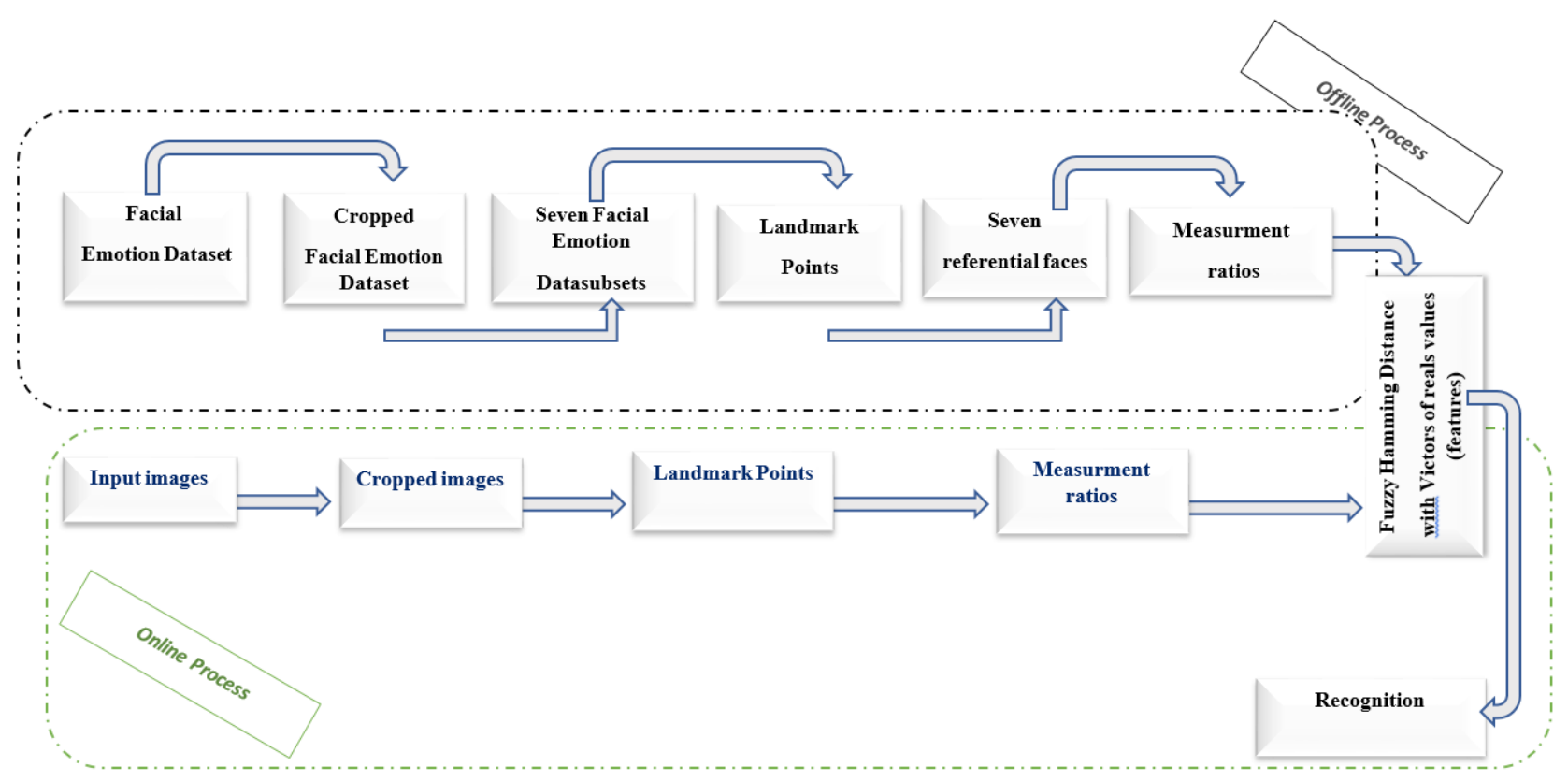

Fig. 3. An overview of our proposed framework based on fuzzy hamming distance with face ratios for facial emotion recognition: Given an input facial emotion: Converted to gray level and cropped to $200 \times 250$ the facial emotion ( $200 \times 250$ is the standard size chosen in the relative database), detected the face and localized 68 landmarks points. Sixteen features are calculated based on the portions of the perfect face and an array of them is generated. These steps are similar between the two modes of facial emotion recognition system FER. In offline process of FER, the system can associate the right kind emotion for each input image: The parameters of Fuzzy Hamming distance are the extracted features from the facial emotion image and the referential faces, on the other side of the system, the generated vector from the facial emotion is involved to recognize its kind of emotion. The output result of our FER is the probe kind of emotion

6. Create the vector:

$\mathrm{V}=\mathrm{xi}, / \mathrm{i} \in\{1, \ldots, 16\}$

7. Compute FHD between Vi (the vector of the input facial emotion photo) and each vector $\mathrm{Vf}$ in the list of referential faces vectors; Their FHD is the same as that between the vector 0 and $|x-y|$. Therefore, the FHD between $x$ and $y$ is the cardinality of the fuzzy Return $\operatorname{setD}(|x-y|, 0)$.

8. Recognition. The output is the kind of emotion of the min distance for the referential face parameter.

\section{Experiments and Results}

To demonstrate the effectiveness of the proposed method, we proceeded for acquiring the dataset of emotional facial expression pictures. We used WSEFEP [11] dataset, the Warsaw Set of Emotional Facial Expression Pictures. The WSEFEP is comprised of those pictures that received the highest recognition marks (e.g., accuracy with intended display) from independent judges, totaling 210 high quality photographs of 30 individuals.

We divide the dataset to seven data subsets suitable for each kind of basics kinds of emotions: Anger, Disgust, Fear, Happiness, Neutral, Sadness and Surprise, Tab. 1 shown the percentage of each kind of emotions.

The WSEFEP is used to training and to testing our approach. Fig. 5 clearly illustrates step by step the results obtained as we progress in the process of the framework already described previously. In the first step the extract emotional photos of the dataset. In the second: The cropped photos.

Tab.1. The percentage of each kind of emotions from WSEFEP dataset

\begin{tabular}{|c|c|c|c|c|c|c|c|}
\hline 节 & 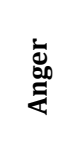 & $\begin{array}{l}\vec{\omega} \\
\stackrel{\vec{b}}{0} \\
\stackrel{0}{0}\end{array}$ & ఫ્屯 & 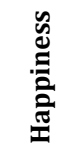 & $\begin{array}{l}\bar{\pi} \\
\bar{E} \\
z\end{array}$ & 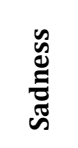 & 芯 \\
\hline$\%$ & 14.28 & 14.28 & 14.28 & 14.28 & 14.28 & 14.28 & 14.28 \\
\hline
\end{tabular}

In the third: The extract 68 face landmarks points. In the fourth: calculate of the sixteen features and in the last step: the vector generated from the facial emotion photos, it is involved to recognized the kind of the facial emotion in the input photo. The output result of FER is the probe kind of emotion.

We generate seven referential faces suitable for each kind of facial emotion based on perfect face ratios and five classical averages: arithmetic mean, geometric mean, harmonic mean, contraharmonic mean and quadratic mean. The result is 35 referential faces. Fig. 4 shown our referential face landmarks photo for each type of average. The images for the usual means are in bleu and they are in red for the author means.

Using our new method, we realized five experiments according to the average used during the generation of the referential face. In each one of them, we 


\begin{tabular}{|c|c|c|c|c|c|}
\hline Emotion & 参 & 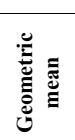 & 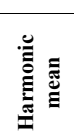 & 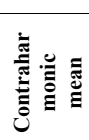 & 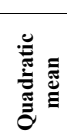 \\
\hline (20) & & & & & \\
\hline$\underbrace{60}_{\text {Disgust }}$ & & & & & \\
\hline$\underset{\text { Fear }}{\%}$ & & & & & \\
\hline$\bigoplus_{\text {Happiness }}$ & & & & & \\
\hline 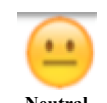 & & & & & \\
\hline 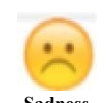 & & & & & \\
\hline$\underset{\text { Surprise }}{\because}$ & & & & & \\
\hline
\end{tabular}

Fig. 4. Our referentiels 68_face emotion_landmarks attributed to their kind of emotion and the kind of mean used to calculate its, each model containbed: "Mouth", "Right_Eyebrow", "Left_Eyebrow", "Right_Eye","Left_Eye", "Nose" and "Jaw".The images for the usuel means are in bleu and they are in red for the auther means

compare the input facial emotion image (features) with the seven referential faces, for that we used the Fuzzy Hamming distance. The output probe kind of emotion is that proper to the referential face having the minimal distance. Tab. 1 shows the cumulative match scores for our approach based on Fuzzy Hamming Distance (FHD) and Average Face Ratios. The result can be considered as a benchmark for the facial emotion recognition system to compare. All exper- imental results of tests are shown in Fig. 6. The cumulative match score is used to evaluate the performance of the algorithms. It measures the percentage of the probe emotion.

Tab. 2 reports the facial emotion recognition accuracies using five different methods: arithmetic mean, geometric mean, harmonic mean, contra harmonic mean and quadratic mean. Our algorithm proves that, the recognition rate reaches more than $63 \%$ for angry 


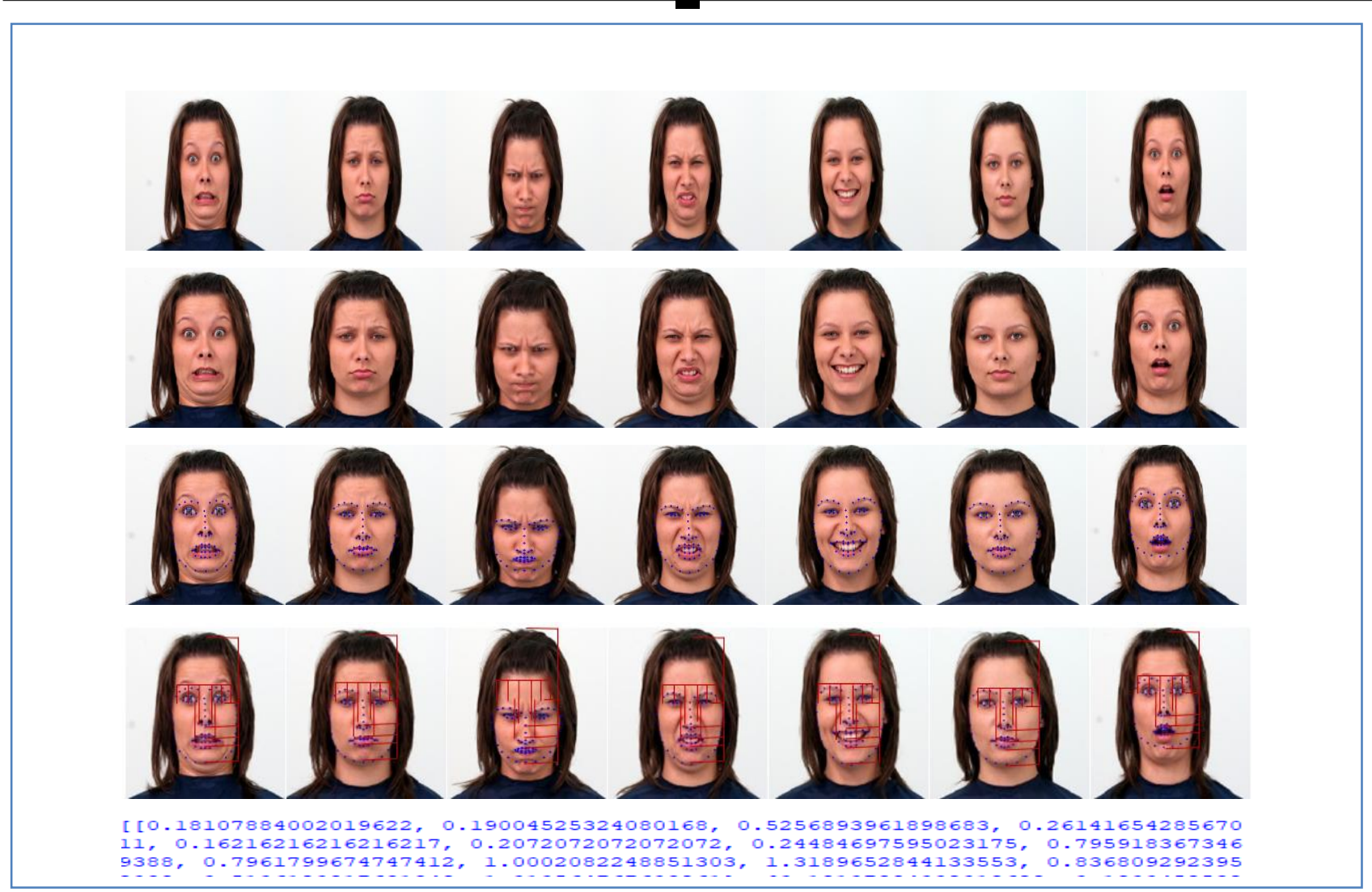

Fig.5. The process of our FSR, line1: extract of the dataset photos/input facial emotion. Line2: cropped emotional photos. Line3: extract the 68 face landmarks points. Line 4: calculate of sixteen features and line5: the vector generated from the facial emotion photos, it is involved to recognize the kind of emotion. The output result of FER is the probe kind of emotion of the input facial photos

emotion, more than $46 \%$ for disgust and fear emotions, more than $93 \%$ for happiness emotion, more than $73 \%$ for neutral emotion, $50 \%$ for sadness emotion and more than $86.67 \%$.

Fig. 6 shows the cumulative match scores for our approach. The $\mathrm{x}$-axis represents the kind of emotion and the $y$-axis represents the recognition rate. The results clearly demonstrate the superiority of our algorithm to recognize the happiness emotion but the last rate of recognition is that of sadness emotion. The quadratic mean helps to better recognize the happiness emotion but not able to better recognize the disgust and sadness emotions. The contraharmonic mean helps to better recognize the angry emotion. The geometric mean helps to better recognize disgust and fear emotions. The arithmetic mean helps to better recognize the sadness emotion and neutral and surprise emotions are recognized with the same rate for the all averages.

The arithmetic mean helps to better recognize the sadness emotion and neutral and surprise emotions are recognized with the same rate for the all averages.

We tested our approach with five different facial emotion recognition methods using classical averages and Fuzzy Hamming distance. In these methods, the cumulative match score proves the performance of the algorithms. The recognition rate reaches more than $93 \%$ in WSEFEP dataset. The algorithmic complexity of our approach is $\mathrm{O}(\mathrm{n})$.

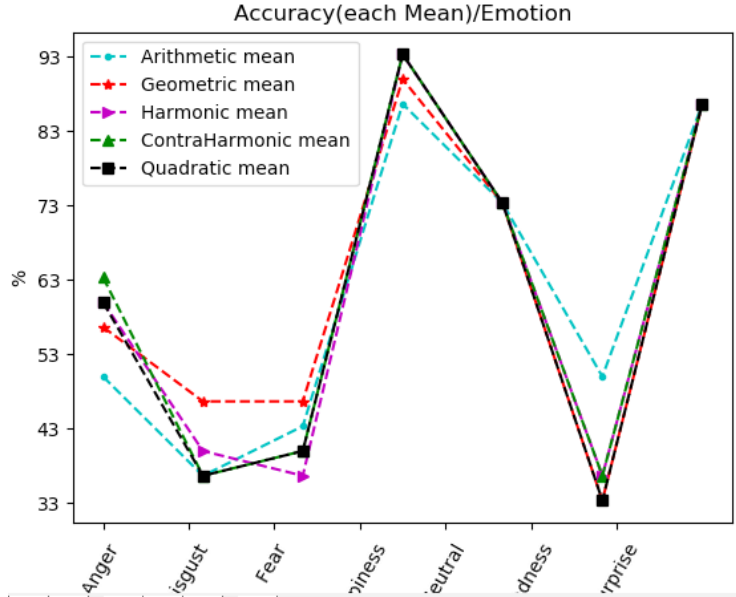

Fig. 6. Comparison of cumulative match scores between our various facial emotion recognition methods using five classical averages

\section{Conclusion}

This paper proposes a new geometrical method for facial emotion recognition. The methods are based on Fuzzy Hamming Distance and the Referential Face Ratios. We used sixteen features based on the distance between different portions of the perfect face. We tested our method on WSEFEP dataset and the results is very satisfactory. Our work is inspired by the recent successful methods that showed that relatively sim- 
ple features could be used to give good performance in a Fuzzy Hamming Distance-based framework. The future work will include a decrease in the number of the features used.

Tab. 2. The cumulative match scores for our approach based on Fuzzy Hamming Distance (FHD) and Average Face Ratios (AFR)

\begin{tabular}{|c|c|c|c|c|c|c|c|}
\hline Emotion & 离 & 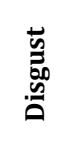 & ఫే & $\frac{\mathscr{d}}{\stackrel{y}{2}}$ & \begin{tabular}{l}
$\overline{3}$ \\
\multirow{2}{0}{} \\
$己$ \\
$z$
\end{tabular} & $\frac{\tilde{d}}{\tilde{\Xi}}$ & 总 \\
\hline $\begin{array}{l}\text { Arithmetic } \\
\text { Mean }\end{array}$ & 50.00 & 36.67 & 43.34 & 86.67 & 73.34 & 50.00 & 86.67 \\
\hline $\begin{array}{l}\text { Geometric } \\
\text { Mean }\end{array}$ & 56.67 & 46.67 & 46.67 & 90.00 & 73.34 & 33.34 & 86.67 \\
\hline $\begin{array}{l}\text { Harmonic } \\
\text { Mean }\end{array}$ & 60.00 & 40.00 & 36.67 & 93.34 & 73.34 & 36.67 & 86.67 \\
\hline $\begin{array}{l}\text { Contra_ } \\
\text { Harmonic } \\
\text { mean }\end{array}$ & 63.34 & 36.67 & 40.00 & 93.34 & 73.34 & 36.67 & 86.67 \\
\hline $\begin{array}{l}\text { Quadratic } \\
\text { Mean }\end{array}$ & 60.00 & 36.67 & 40.00 & 93.34 & 73.34 & 33.34 & 86.67 \\
\hline
\end{tabular}

\section{AUTHORS}

Khalid Ounachad* - Department of Informatics, Faculty of Sciences, Ibn Tofail University, Kenitra, Morocco, e-mail: khalid.ounachad@uit.ac.ma.

Mohamed Oualla - Department of Informatics, Faculty of Sciences, Ibn Tofail University, Kenitra, Morocco, e-mail: mohamedoualla76@gmail.com.

Abdelalim Sadiq - Department of Informatics, Faculty of Sciences, Ibn Tofail University, Kenitra, Morocco, e-mail: a.sadiq@uit.ac.ma.

Abdelghani Souhar, Department of Informatics, Faculty of Sciences, Ibn Tofail University, Kenitra, Morocco, e-mail: houssouhar@gmail.com.

*Corresponding author

\section{REFERENCES}

[1] P. Ekman, W. V. Friesen, M. O’Sullivan, A. Chan, I. Diacoyanni-Tarlatzis, K. Heider, R. Krause, W. A. LeCompte, T. Pitcairn and P. E. Ricci-Bitti, "Universals and cultural differences in the judgments of facial expressions of emotion", Journal of Personality and Social Psychology, vol. 53, no. 4, 1987, 712-717, DOI: $10.1037 / / 0022-3514.53 .4 .712$.

[2] A. Bookstein, S. T. Klein and T. Raita, "Fuzzy Hamming Distance: A New Dissimilarity Measure". In: G. M. Landau and A. Amir (eds.), Combinatorial Pattern Matching, 2001, 86-97, DOI: 10.1007/3-540-48194-X_7.
[3] K. Ounachad, A. Sadiq and A. Souhar, "Fuzzy Hamming Distance and Perfect Face Ratios Based Face Sketch Recognition". In: 2018 IEEE 5th International Congress on Information Science and Technology (CiSt), 2018, 317-322, DOI: $10.1109 /$ CIST.2018.8596665.

[4] M. Ionescu and A. Ralescu, "Fuzzy Hamming Distance Based Banknote Validator". In: The 14th IEEE International Conference on Fuzzy Systems, 2005. FUZZ '05, 2005, 300-305, DOI: 10.1109/FUZZY.2005.1452410.

[5] M. Ionescu, "Image clustering for a fuzzy hamming distance based cbir system". In: Proceedings of the Sixteen Midwest Artificial Intelligence and Cognitive Science Conference, 2005, 102-108.

[6] M. Ionescu and A. Ralescu, "Fuzzy hamming distance in a content-based image retrieval system". In: 2004 IEEE International Conference on Fuzzy Systems, vol. 3, 2004, 1721-1726, DOI: $10.1109 /$ FUZZY.2004.1375443.

[7] G. S. Shehu, A. M. Ashir and A. Eleyan, "Character recognition using correlation hamming distance". In: 2015 23nd Signal Processing and Communications Applications Conference (SIU), 2015, 755-758,

DOI: $10.1109 /$ SIU.2015.7129937.

[8] S. Xiao, S. Yan, and A. A. Kassim, "Facial Landmark Detection via Progressive Initialization". In: 2015 IEEE International Conference on Computer Vision Workshop (ICCVW), 2016, 986993,

DOI: 10.1109/ICCVW.2015.130.

[9] K. Ounachad, M. Oualla, A. Souhar and A. Sadiq, "Structured learning and prediction in face sketch gender classification and recognition", International Journal of Computational Vision and Robotics, vol. 10, no. 6, 2020, DOI: 10.1504/IJCVR.2020.110645.

[10] R. Chellappa, C. L. Wilson and S. Sirohey, "Human and machine recognition of faces: a survey", Proceedings of the IEEE, vol. 83, no. 5, 1995, 705-741,

DOI: $10.1109 / 5.381842$.

[11] M. Olszanowski, G. Pochwatko, K. Kuklinski, M. Scibor-Rylski, P. Lewinski and R. K. Ohme, "Warsaw set of emotional facial expression pictures: a validation study of facial display photographs", Frontiers in Psychology, vol. 5, 2015, DOI: 10.3389/fpsyg.2014.01516. 